\title{
Boundary value problems of elasticity theory for plane domains with one-dimensional elastic reinforcements
}

\author{
S. E. Mikhailov and I. V. Namestnikova
}

Many authors have examined problems related to the load transmission from an elastic rod to an elastic plane. It was assumed in the majority of investigations that the stringer is a thin rectilinear rod transmitting only longitudinal forces while the rod contact with the plane is realized along a line. Different modifications of sheet contact with a rectilinear tensile stringer considered as an inner stringer of finite length or as an infinite edge stringer were analyzed in [1,2]. Problems about the reinforcement of holes in a plate by a thin rod of constant section that possesses bending and longitudinal stiffnesses were solved in [3]. The eccentricity of the connection between the shell middle surface and the rod was taken into account in [4] in a study of shells reinforced by thin curvilinear rods. other models of the one-dimensional element connected to an elastic medium without taking account of its bending stiffness were analyzed in $[5,6]$. Solutions of a number of problems with circular reinforcing elements are obtained in [7].

An isotropic finite or infinite, linearly elastic plate reinforced along part or all of the boundary and along certain internal lines by elastic curvilinear rods possessing variable longitudinal and bending stiffnesses, variable curvature and thickness, the eccentricity of the connection to the plate and with an arbitrary transverse section shape symmetric relative to the plate middle surface are studied in this paper. Boundary conditions on the line of plate contact with the inner or edge elastic rods are obtained for the reinforcement models generalizing $[1,2]$ by using the theory of elastic rods in the case of a plane state of stress. Existence and uniqueness theorems are proved for appropriate boundary value problems; the singularity of the stresses at angles and tips of the rods are proved. The relationships obtained carry over completely to the plane strain problem for an elastic cylinder reinforced by homogeneous cylindrical shells along the generator. Some of the results described here are represented in [8].

\section{ONE-DIMENSIONAL CURVILINEAR REINFORCEMENTS}

Let $x_{i}$ be a Cartesian coordinate system whose plane $\left(x_{1}, x_{2}\right)$ agrees with the plane of the rod central axis $\mathrm{L}_{0}$ (the line of centers of gravity of its transverse sections) that is a plane piecewise-smooth curve without reentry points, and let $x_{i}{ }^{0}$ be coordinates of the points of $L_{0}$. Let us parametrize the central axis by using the arc length $\tau$, and let us denote the rod transverse section by $\Omega(\tau)$, the boundary of the transverse section by $\partial \Omega(\tau)$, and its area by $\mathrm{A}(\tau)$. Furthermore, unless specified otherwise, we consider the subscripts on the subscripted parameters to vary between 1 and 2 and understand summation between these limits over repeated subscripts. Let us introduce the vector tangent to the central axis $\mathrm{k}_{i}{ }^{0}=\dot{\mathrm{x}}_{i}{ }^{0}(\tau)$ and its normal $\mathrm{n}_{i}{ }^{0}(\tau)=\mathrm{e}_{i j} \mathrm{k}_{j}{ }^{0}(\tau)$, where $e_{i j}$ is an alternating symbol: $e_{12}=$ $-e_{21}=1, e_{11}=e_{22}=0$, and the dot overhead denotes the derivative with respect to $\tau$. We assume one of the principal axes of inertia of the rod transverse section lies in the $\left(x_{1}\right.$, $\mathrm{x}_{2}$ ) plane.

Let bulk forces $\hat{f}_{i}(x)$ act on the rod, while an external surface load $\hat{T}_{1}(x)^{\prime} F_{i}(\tau)$ is applied to the rod side surface, $\tau_{0}$ is the principal vector of forces in the transverse section of the rod $\tau<\tau_{0}$ acting on its part with coordinates $\tau>\tau_{0}$ from the side of the part $M\left(\tau_{0}\right)$ is the moment of these forces relative to the center of the gravity of the transverse section (the moment acting counterclockwise is considered positive), $v_{i}{ }^{0}(\tau)$ is the displacement of the rod central axis. We designate the nodes $\tau_{R}(\alpha)\left(\alpha=1-N_{R}\right)$ of the line $L_{0}$ its angular points, where we will consider conditions of rigid connection of parts of the rod given and also points where concentrated (on sections passing through these points) forces and moments are given. 
Let the boundary conditions

$$
F_{i}\left(\tau_{1}\right)=-F_{i}^{(1)}, M\left(\tau_{1}\right)=-M^{(1)}, F_{i}\left(\tau_{2}\right)=F_{i}^{(2)}, M\left(\tau_{2}\right)=M^{(2)}
$$

be given on the ends of the $\operatorname{rod} \tau_{1}, \tau_{2}\left(\tau_{2}>\tau_{1}\right)$. If the rod is closed, we select a point $\tau_{1}$ such that no forces or moments would be concentrated there, then the boundary conditions (1.1) are replaced there by continuity conditions for the forces, moments, displacements, and rotations

$$
\begin{gathered}
\left.F_{i}(\tau)\right|_{\tau_{1}} ^{\tau_{2}}=\left.M(\tau)\right|_{\tau_{1}} ^{\tau_{2}}=0, \tau_{2}=\tau_{1}+l \\
\left.v_{i}^{0}(\tau)\right|_{\tau_{1}} ^{\tau_{2}}=\left.\dot{v}_{i}^{0}(\tau) n_{i}^{0}(\tau)\right|_{\tau_{1}} ^{\tau_{2}}=0 .
\end{gathered}
$$

We have at the nodes $\tau_{R}(\alpha)$

$$
\begin{gathered}
F_{i}\left(\tau_{R}^{(\alpha)}+0\right)-F_{i}\left(\tau_{R}^{(\alpha)}-0\right)=-F_{R i}^{(\alpha)}, M\left(\tau_{R}^{(\alpha)}+0\right)-M\left(\tau_{R}^{(\alpha)}-0\right)=-M_{R}^{(\alpha)}, \\
v_{i}^{0}\left(\tau_{R}^{(\alpha)}+0\right)-v_{i}^{0}\left(\tau_{R}^{(\alpha)}-0\right)=0, \\
\dot{v}_{i}^{0}\left(\tau_{R}^{(\alpha)}+0\right) n_{i}^{0}\left(\tau_{R}^{(\alpha)}+0\right)-\dot{v}_{i}^{0}\left(\tau_{R}^{(\alpha)}-0\right) n_{i}^{0}\left(\tau_{R}^{(\alpha)}-0\right)=0 .
\end{gathered}
$$

Here $F_{i}(j), F_{R i}(\alpha)$ are given forces, $M(j), M_{R}(\alpha)$ are given bending moments, and $\ell$ is the length of $\mathrm{L}_{0}, j=1,2$.

Let $\xi=\left(x_{i}-x_{i}{ }^{0}(\tau)\right) n_{i}{ }^{0}(\tau)$ be the projection of the radius-vector connecting the center of gravity of the transverse section with an arbitrary point of the section on the normal $n_{j}{ }^{0}(\tau)$. Using the hypothesis of plane sections, we represent the displacement of a point of the rod that does not lie on the central axis with coordinates $x_{i}(\tau, \xi)=x_{i}(\tau, 0)+\xi n_{i}{ }^{0}(\tau)$ [9]

$$
\begin{gathered}
v_{i}(\tau, \xi):=x_{i}^{\prime}(\tau, \xi)-x_{i}(\tau, \xi)=v_{i}^{0}(\tau)-\xi h_{i}^{0} n_{j}^{0} v_{j}^{0}(\tau), \\
v_{i}^{0}(\tau):=v_{i}(\tau, 0), x_{i}^{0}(\tau):=x_{i}(\tau, 0)
\end{gathered}
$$

( $\mathrm{x}_{\mathrm{i}}$ and $\mathrm{x}_{1}$ ' are coordinates of a point before and after deformation). It is taken into account in deriving (1.5) that under conditions of smallness of the angles of rotation

$$
n_{i}^{0^{\prime}}=e_{i j} \frac{d x_{j}^{0^{\prime}} d \tau}{d \tau d \tau^{\prime}} \approx e_{i \beta}\left(k_{\beta}^{0}+\dot{v}_{\beta}^{0}\right)\left(1-k_{j}^{0} v_{j}^{0}\right) \approx n_{i}^{0}-k_{i}^{0} \dot{v}_{j}^{0}(\tau) n_{j}^{0}(\tau)
$$

Neglecting transverse stesses in the rod, setting $|\dot{\xi}| \ll 1$ and using the Hooke's law, after integrating over $\Omega$ we express the longitudinal force $F_{i}(\tau) k_{i}{ }^{0}(\tau)$ acting in the transverse section of the rod and the bending moment $M(\tau)$ relative to the center of gravity in terms of the displacement of the central axis

$$
\begin{gathered}
F_{i}(\tau) k_{i}^{0}(\tau)=\left(G+\chi^{2} G_{1}\right) \dot{v}_{i}^{0} k_{i}^{0}+\chi^{G}\left(n_{i}^{0} \dot{v}_{i}^{0}\right)^{*}, \\
M(\tau)=-G_{1}\left[\chi_{i}^{0} k_{i}^{0}+\left(n_{i}^{0} \dot{v}_{i}^{0}\right)^{\cdot}\right], \\
G(\tau)=E^{(r)}(\tau) A(\tau), G_{1}(\tau)=E^{(r)}(\tau) J(\tau), \quad J(\tau)=\int_{\Omega(\tau)} \xi^{2}(1+\chi(\tau) \xi)^{-1} d \Omega .
\end{gathered}
$$

Here $\chi(\tau)=k_{i}{ }^{0} \dot{n}_{j}{ }^{0}$ is the curvature of the rod central axis ( $\chi>0$ if the center of the tangent circle lies to the left of the rod during a positive transversal), $E(r)$ is the Young's modulus of the rod material, and $G$ and $G_{1}$ are the rod longitudinal and bending stiffnesses.

The rod equilibrium equations in the notation used are written in the form $[4,9]$

$$
\begin{gathered}
\dot{F}_{i}(\tau)=-p_{i}(\tau), \dot{M}(\tau)-F_{i}(\tau) n_{i}^{0}(\tau)=-m(\tau) ; \\
p_{i}(\tau)=\iint_{\Omega(\tau)} \hat{f}_{i} \vartheta(\tau, \xi) d \Omega+\int_{\partial \dot{Q}(\tau)} \widehat{T}_{i} \vartheta(\tau, \xi) d \Gamma,
\end{gathered}
$$




$$
\begin{gathered}
m(\tau)=\int_{\Omega(\tau)} \int_{\hat{f}_{i}} k_{i} \xi \vartheta(\tau, \xi) d \Omega+\int_{\partial \Omega(\tau)} \widehat{T}_{i} k_{i \xi}^{0} \xi(\tau, \xi) d \Gamma, \\
\theta(\tau, \xi):=\left\{[1+\chi(\tau) \xi(\tau)]^{2}+\xi^{2}(\tau)\right\}^{1 / 2},
\end{gathered}
$$

where $p_{i}(\tau)$ and $m(\tau)$ are the linear forces and moments that occur during transfer of the bulk load $\hat{f}_{i}$ and surface load $\hat{\mathrm{T}}_{i}$ acting on the rod over the line $\mathrm{L}_{0}$, and are distributed along the rod central axis. Together with the boundary conditions (1.1)-(1.4) the relationships (1.6) and (1.7) yield a complete system of equations for plane curvilinear rods. Furthermore, rods are examined for which the longitudinal and bending stiffnesses are $0<G$, $\mathrm{G}_{1} \leq \infty$ while the curvature is $|\chi|<\infty$.

We shall consider the set of functions $\left\{v_{i}{ }^{0}(\tau), F_{j}(\tau), M(\tau)\right\}$ on the line $L_{0}$ to belong to the class $H^{(r)}\left(L_{0}\right)$ if the functions $v_{i}{ }^{0}(\tau), \dot{v}_{i}{ }^{0}(\tau) n_{i}{ }^{0}(\tau), F_{i}(\tau), M(\tau)$ are absolutely continuous at all points of $\mathrm{L}_{0}$ with the exception, perhaps, of the nodal points, satisfy (1.6) almost everywhere on $\mathrm{L}_{0}$, and have finite left and right limits at the nodes.

Let the set $\left\{v_{i}{ }^{0}, F_{i}, M\right\},\left\{v_{i}^{* 0}, F_{i}^{*}, M^{*}\right\} \in H^{(r)}$. We introduce the notation

$$
\begin{aligned}
2\left\langle v^{0}, v^{* 0}\right\rangle^{(r)}:= & \int_{\dot{L}_{0}}\left\{G \dot{G}_{i}^{0} k_{i}^{0} \dot{v}_{j}^{* 0} k_{j}^{0}+G_{1}\left[\chi \dot{v}_{i}^{0} k_{i}^{0}+\left(n_{i}^{0} v_{i}^{0}\right)^{0}\right]\left[\chi_{j}^{*} \dot{v}_{j}^{* 0} k_{j}^{0}+\left(n_{j}^{0} \dot{v}_{j}^{* 0}\right)^{j}\right]\right\} d \tau= \\
& \int_{L_{0}}\left\{\frac{1}{G}\left(F_{i} k_{i}^{0}+\chi M\right)\left(F_{j}^{*} k_{j}^{0}+\chi M^{*}\right)+\frac{1}{G_{1}} M M^{*}\right\} d \tau .
\end{aligned}
$$

Then setting $\mathrm{P}_{i}=-\dot{\mathrm{F}}_{i}, \mathrm{~m}=-\dot{M}+\mathrm{F}_{i} \mathrm{n}_{\dot{1}}{ }^{0}$, by using integration by parts we obtain the Green's formula for the rod

$$
\begin{gathered}
2\left\langle v^{0}, v^{* 0}\right\rangle^{(r)}=\int_{L_{0}}\left(p_{i} v_{i}^{* 0}-m \dot{v}_{i}^{* 0} n_{i}^{0}\right) d \tau+ \\
+\left[F_{i}(\tau) v_{i}^{* 0}(\tau)-M(\tau) \dot{v}_{i}^{* 0}(\tau) n_{i}^{0}\left(\tau^{0}\right)\right]_{\tau_{1}}^{\tau_{2}}- \\
-\sum_{\alpha=1}^{N_{R}}\left[F_{i}(\tau) v_{i}^{* 0}(\tau)-M(\tau) \dot{v}_{i}^{0}(\tau) n_{i}^{0}(\tau)\right]_{\tau_{R}^{(\alpha)}-0^{\prime}}^{\tau_{R}^{(\alpha)}+0}
\end{gathered}
$$

where the sum is taken over all nodal points $\tau_{R}(\alpha)$ and the components for the tips $\tau_{1}, \tau_{2}$ drop out if the rod is closed. Taking into account that the rod elastic energy is $\left\langle\mathrm{v}^{0}\right.$, $\mathrm{v}^{0}>(\mathrm{r}) \geq 0$ because of $(1.9)$, it is easy to prove a uniqueness theorem for the rod: the solution of the problem $(1.6),(1.7),(1.1)-(1.4)$ in the class $H(r)$ is unique to the accuracy of a rigid shift with the rotation $v_{C_{i}}{ }^{0}(\tau)=C_{i}+C_{3} e_{i j} x_{j}{ }^{0}(\tau)$.

Setting $v_{i} *^{0}=C_{i}$ or $v_{i} *^{0}=e_{i j} x_{j}^{0}$ in (1.9), we also have that it is necessary for the existence of a solution to the problem $(1.6),(1.7),(1.1)-(1.4)$ in $H^{(r)}$ that the sums of the forces and moments applied to the rod be zero

$$
\begin{gathered}
\int_{L_{0}} p_{i}(\tau) d \tau+F_{i}^{(1)}+F_{i}^{(2)}+\sum_{\alpha=1}^{N_{R}} F_{R i}^{(\alpha)}=0 \\
\int_{\dot{L}_{0}}\left[p_{i}(\tau) e_{i j} x_{j}^{0}(\tau)-m(\tau)\right] d \tau+F_{i}^{(1)} e_{i j} x_{j}^{0}(\tau)+F_{i}^{(2)} e_{i j} x_{j}^{0}\left(\tau_{2}\right)-M^{(1)}-M^{(2)}+\sum_{\alpha=1}^{N_{R}}\left[F_{R i}^{(\alpha)} e_{i j} x_{j}^{0}\left(\tau_{R}^{(\alpha)}\right)-M_{R}^{(\alpha)}\right]=0 .
\end{gathered}
$$

Because of (1.2) the tip terms $F_{i}(j), M(j), j=1,2$ drop out of these relations for a closed rod.

The relationships (1.6) and (1.7) can be integrated, then

$$
\begin{gathered}
v_{i}^{0}=v_{0 i}^{0}+C_{i}+C_{3} e_{i j} x_{j}^{0}(\tau), v_{0 i}^{0}(\tau ; p)= \\
=\int_{\tau_{1}}^{\tau}\left\{[g(\eta) N(\eta) \div a(\eta) M(\eta)] k_{i}^{0}(\eta)-n_{i}^{0}(\eta) \int_{\tau_{1}}^{\eta}\left[a\left(\eta^{0}\right) N\left(\eta^{0}\right)+b\left(\eta^{0}\right) M\left(\eta^{0}\right)\right]\right\} d \eta
\end{gathered}
$$




$$
\begin{array}{r}
N\left(\eta^{0} ; p\right):=F_{i}\left(\eta^{0} ; p\right) k_{i}^{0}\left(\eta^{0}\right), F_{i}\left(\eta^{0} ; p\right)=-\int_{\tau_{1}}^{\eta_{1}^{0}} p_{i}(t) d t+F_{i}\left(\tau_{1} ; p\right)- \\
-\sum_{\tau_{R}^{(\alpha)} \leqslant \eta^{0}} F_{R i}^{(\alpha)}, M\left(\eta^{0} ; p\right):=-\int_{\tau_{1}}^{\eta_{1}^{0}} n_{i}^{(0)}\left(\tau^{V}\right) d \tau \vee \int_{\tau_{1}}^{\tau^{V}} p_{i}(t) d t-\int_{\tau_{1}}^{\eta^{0}} m(t) d t+ \\
+F_{i}\left(\tau_{1} ; p\right) y_{i}^{1}\left(\eta^{0}\right)+M\left(\tau_{1} ; p\right)-\sum_{\tau_{R}^{(\alpha)} \leqslant \eta^{0}}\left\{\left[y_{i}^{1}\left(\eta^{0}\right)-y_{i}^{1}\left(\tau_{R}^{(\alpha)}\right)\right] F_{R i}^{(\alpha)}+M_{R}^{(\alpha)}\right\} .
\end{array}
$$

Here and henceforth $y_{i}{ }^{1}\left(\eta^{0}\right)=e_{i j}\left[x_{j}{ }^{0}\left(\eta^{0}\right)-x_{j}{ }^{0}\left(\tau_{1}\right)\right] ; a\left(\eta^{0}\right)=\chi\left(\eta^{0}\right) / G\left(\eta^{0}\right) ; b\left(\eta^{0}\right)=1 / G_{1}\left(\eta^{0}\right)+$ $x^{2}\left(\eta^{0}\right) / G\left(\eta^{0}\right) ; g\left(\eta^{0}\right)=1 / G\left(\eta^{0}\right)$ and the set of external loads for the rod $\left\{\mathrm{p}_{i}, \mathrm{~m}, \mathrm{~F}_{\mathrm{Ri}}(\alpha)\right.$, $\left.M_{R}(\alpha)\right\}$ is denoted by the symbol $p$ without subscripts. The constants $C_{\alpha}(\alpha=1-3)$ in (1.13) are arbitrary by virtue of the uniqueness theorem. Conditions on the jump (1.4) were taken into account to obtain (1.14).

It is also necessary to put $F_{i}\left(\tau_{1} ; p\right)=-F_{i}(1), M\left(\tau_{1} ; p\right)=-M(1)$ to satisfy the first pair of conditions (1.1) at the ends of an open rod. As is easily seen, the second pair of conditions in (1.1) reduces to the solvability conditions (1.11) and (1.12) of the problem for a rod in the original formulation (1.6), (1.7), (1.1)-(1.4).

The relationships (1.13) and (1.14) can be rewritten in the form

$$
\begin{gathered}
v_{0 i}^{0}(\tau)(\tau ; p)=\int_{\tau_{i}}^{\tau}\left[\left[\Pi_{i j}^{1} p_{j}+\Pi_{i}^{(1 m)} m\right]\left(\tau_{0}\right)+\Pi_{i j}^{1}\left(\tau_{0}, \tau_{i}\right) F_{j}^{(1)}+\Pi_{i}^{(1 m)}\left(\tau_{0}, \tau_{1}\right) M^{(1)}+\right. \\
\left.+\sum_{\alpha=1}^{\alpha_{R}}\left[\Pi_{i j}^{1}\left(\tau_{0}, \tau_{R}^{(\alpha)}\right) F_{R j}^{(\alpha)}+\Pi_{i}^{(1 m)}\left(\tau_{0}, \tau_{R}^{(\alpha)}\right) M_{R}^{(\alpha)}\right] H\left(\tau_{0}-\tau_{R}^{(\alpha)}\right)\right\} d \tau_{0}
\end{gathered}
$$

where $H(\tau)$ is the Heaviside function and $\Pi_{i j}^{1}, \Pi_{i}^{(1 m)}$ are Volterra operators

$$
\begin{gathered}
{\left[\Pi_{i j}^{1} p_{j}\right]\left(\tau_{0}\right)=\int_{\tau_{1}}^{\tau_{0}} \Pi_{i j}^{1}\left(\tau_{0}, t\right) p_{j}(t) d t,\left[\Pi_{i}^{(1 m)} m\right]\left(\tau_{0}\right)=\int_{\tau_{i}}^{\tau_{0}} \Pi_{i}^{(\mathrm{lm})}\left(\tau_{0}, t\right) m(t) d t,} \\
\Pi_{i j}^{1}\left(\tau_{0}, t\right)=-k_{i}^{0}\left(\tau_{0}\right)\left[k_{j}^{0}\left(\tau_{0}\right) g\left(\tau_{0}\right)-a\left(\tau_{0}\right)\left(y_{j}^{1}(t)-y_{j}^{1}\left(\tau_{0}\right)\right)\right]+ \\
\quad+n_{i}^{0}\left(\tau_{0}\right)\left[E_{j}\left(\tau_{0}\right)-E_{j}(t)-y_{j}(t)\left(B\left(\tau_{0}\right)-B(t)\right)\right] \\
\Pi_{i}^{(1 m)}\left(\tau_{0}, t\right)=-k_{i}^{0}\left(\tau_{0}\right) a\left(\tau_{0}\right)+n_{i}^{0}\left(\tau_{0}\right)\left(B\left(\tau_{0}\right)-B(t)\right) .
\end{gathered}
$$

Here the functions $E_{i}, B$ are determined in terms of the rod stiffness and geometry

$$
B(\tau)=\int_{\tau_{1}}^{\tau} b\left(\eta^{0}\right) d \eta^{0}, E_{i}(\tau)=\int_{\tau_{1}}^{\tau}\left\{a\left(\eta^{0}\right)\left[k_{i}^{0}\left(\eta^{0}\right)+\chi\left(\eta^{0}\right) y_{i}^{1}\left(\eta^{0}\right)\right]+y_{i}^{1}\left(\eta^{0}\right) / G_{1}\left(\eta^{0}\right)\right\} d \eta^{0} .
$$

After substituting (1.14) into the force and moment continuity conditions (1.2) for a closed rod, we arrive at the solvability conditions (1.11), (1.12), just as for the open rod (if the end terms are discarded there). If the stiffnesses are $0<\left(G(\tau), G_{1}(\tau)\right)<\infty$ at least at some part of the curve $L_{0}$, then by taking account of the Green's formula (1.10) it can be shown that the continuity conditions for the displacements and angles of rotation (1.3), not utilized up to now, are necessary and sufficient for determination of the three unknown constants $F_{i}\left(\tau_{1} ; p\right)$ and $M\left(\tau_{1}, p\right)$ in the form of functionals of $p_{i}$ and $m$. Substituting these values for the constants into (1.15), we obtain

$$
\begin{gathered}
v_{0 i}(\tau ; p)=\int_{\tau_{1}}^{\tau}\left\{\left[\Pi_{i j} p_{j}+\Pi_{i}^{(m)} m\right]\left(\tau_{0}\right)+\sum_{\alpha=1}^{N_{R}}\left[\left[\Pi_{i j}^{1}\left(\tau_{0}, \tau_{R}^{(\alpha)}\right) F_{R j}^{(\alpha)}+\right.\right.\right. \\
\left.\left.\left.+\Pi_{i}^{(1 m)}\left(\tau_{0}, \tau_{R}^{(\alpha)}\right) M M_{R}^{(\alpha)}\right] H\left(\tau_{0}-\tau_{R}^{(\alpha)}\right)+\Pi_{i j}^{0}\left(\tau_{0}, \tau_{R}^{(\alpha)}\right) F_{R j}^{(\alpha)}+\Pi_{i}^{(0 m)}\left(\tau_{0}, \tau_{R}^{(\alpha)}\right) M_{R}^{(\alpha)}\right]\right\} d \tau_{0},
\end{gathered}
$$

where the operators $\Pi_{i, j}, \Pi_{i}^{(m)}$ are sums of the Volterra operators $\Pi_{i j}^{1}, \Pi_{i j}^{(1 m)}$ represented in 
(1.16) and finite-dimensional (degenerate) operators

$$
\begin{gathered}
{\left[\Pi_{i j} p_{j}\right]\left(\tau_{0}\right)=\left[\Pi_{i j}^{1} p_{j}\right]\left(\tau_{0}\right)+\int_{L_{0}} \Pi_{i j}^{0}\left(\tau_{0}, t\right) p_{j}(t) d t,} \\
{\left[\Pi_{i j}^{(m)} m\right]\left(\tau_{0}\right)=\left[\Pi_{i j}^{(1 m)} m\right]\left(\tau_{0}\right)+\int_{L_{0}} \Pi_{i j}^{(o m)}\left(\tau_{0}, t\right) m(t) d t,} \\
\Pi_{i j}^{0}\left(\tau_{0}, t\right)=-k_{i}^{0}\left(\tau_{0}\right)\left[-k_{\alpha}^{0}(\tau) \eta_{\alpha j}(t) g\left(\tau_{0}\right)-a\left(\tau_{0}\right)\left(\zeta_{j}(t)+y_{\alpha}^{1}\left(\tau_{0}\right) \eta_{\alpha j}(t)\right)\right]+ \\
+n_{i}^{0}\left(\tau_{0}\right)\left[-\eta_{\alpha j}(t) E_{\alpha}\left(\tau_{0}\right)-\zeta_{j}(t) B\left(\tau_{0}\right)\right], \\
\Pi_{i}^{(0 m)}\left(\tau_{0}, t\right)=-k_{i}^{0}\left(\tau_{0}\right)\left[-k_{\alpha}^{0}\left(\tau_{0}\right) \eta_{\alpha}^{(m)}(t) g\left(\tau_{0}\right)-a\left(\tau_{0}\right)\left(\zeta^{(m)}(t)+\right.\right. \\
\left.\left.+y_{\alpha}^{1}\left(\tau_{0}\right) \eta_{\alpha}^{(m)}(t)\right)\right]+n_{i}^{0}\left(\tau_{0}\right)\left[-\eta_{\alpha}^{(m)}(t) E_{\alpha}\left(\tau_{0}\right)-\zeta^{(m)}(t) B\left(\tau_{0}\right)\right] .
\end{gathered}
$$

The functions $\eta_{\alpha j}, \zeta_{j}, \eta_{\alpha}^{(m)}, \zeta^{(m)}$ are determined explicitly in terms of the rod curvature and geometry

$$
\begin{aligned}
& B^{0}=B\left(\tau_{1}+l\right), E_{i}^{0}=E_{i}\left(\tau_{1}+l\right), B^{V}(\tau)=B^{0}-B(\tau), E_{i}^{\bigvee}(\tau)=E_{i}^{0}-E_{i}(\tau), \\
& \zeta_{j}(\tau)=\left[E_{j}^{\bigvee}(\tau)-y_{j}^{1}(\tau) B^{V}(\tau)-E_{i}^{0} \eta_{i j}(\tau)\right] / B^{0}, \eta_{i j}(\tau)=\left(\Omega^{-1}\right)_{i k} \omega_{k j}(\tau), \\
& \left(\Omega^{-1}\right)_{i k}=\left(\delta_{i k} \Omega_{\alpha \alpha}-\Omega_{i k}\right) / \Delta^{\wedge}, \Delta^{\wedge}=\Omega_{11} \Omega_{22}-\Omega_{12} \Omega_{21}, \Omega_{i j}=I_{i j}^{0} B^{0}-E_{i}^{0} E_{j}^{0}, \\
& \omega_{i j}(\tau)=\left[I_{i j}^{\vee}(\tau)-y_{j}^{1}(\tau) E_{i}^{V}(\tau)\right] B^{0}-\left[E_{j}(\tau)-y_{j}^{1}(\tau) B^{V}(\tau)\right] E_{i}^{0}, \\
& I_{i j}(\tau)=\int_{\tau_{1}}^{\tau}\left(g\left(\eta^{0}\right)\left[k_{i}^{0}\left(\eta^{0}\right)+\chi\left(\eta^{0}\right) y_{i}\left(\eta^{0}\right)\right]\left[k_{j}^{0}\left(\eta^{0}\right)+\chi\left(\eta^{0}\right) y_{j}^{1}\left(\eta^{0}\right)\right]+\right. \\
& \left.+\left[1 G_{1}\left(\eta^{0}\right)\right] y_{j}^{1}\left(\eta^{0}\right) y_{j}^{1}\left(\eta^{0}\right)\right] d \eta^{0}, \\
& I_{i j}^{0}=I_{i j}\left(\tau_{1}+l\right), I_{i j}^{Y}(\tau)=I_{i j}^{0}-I_{i j}(\tau), \eta_{i}^{(m)}(\tau)=\left(\Omega^{-1}\right)_{i k}\left(0_{0}^{(m)}(\tau),\right. \\
& \zeta^{(m)}(\tau)=\left[E_{i}^{0} \eta_{i}^{(m)}(\tau)-B^{\bigvee}(\tau)\right] / B^{a}, \omega_{i}^{(m)}(\tau)=E_{i}^{\bigvee}(\tau) B^{0}-E_{i}^{0} B^{\bigvee}(\tau) .
\end{aligned}
$$

As already mentioned, by virtue of the Green's formula the relationships (1.19) yield bounded kernels if $\Pi_{i j}{ }^{0}, \Pi_{i j}(0 \mathrm{~m})$ at some part of the curve $L_{0} 0<\left(G(\tau), G_{1}(\tau)\right)<\infty$. When $G(\tau)=G_{1}(\tau)=\infty$ on the whole rod, it is necessary to set $v_{0 i}=0$. And when either the longitudinal stiffness is $G(\tau)=\infty$ or the bending stiffness is $G_{1}(\tau)=\infty$ at each point of $L_{0}$ the conditions (1.3) can already not be necessary and sufficient to determine the forces $F_{j}\left(\tau_{1}\right.$; p) and moments $M\left(\tau_{1} ; p\right)$, however the displacements $v_{i}{ }^{0}$ will nevertheless be determined and unique to the accuracy of constants $C_{i}$ and the functions $v_{i 0}{ }^{0}$ are representable in the form (1.17), where the kernels $\Pi_{i j}{ }^{0}, \Pi_{i j}{ }^{(0 \mathrm{~m})}$ can be obtained by passing to the limit from (1.18) and (1.19).

The equations presented in this section and the reasoning are carried over also to cylindrical shells homogeneous along the generators that are under plane deformation conditions if $G(\tau)=E(\tau) 2 h(\tau) /\left(1-\nu^{2}(\tau)\right)$ is the linear longitudinal stiffness, $G_{1}(\tau)=E(\tau)[2 h(\tau)]^{3} /$ $\left[12\left(1-v^{2}(\tau)\right)\right]$ is the linear bending stiffness of the shell and are set in (1.6) below (E, $\nu$, and $2 \mathrm{~h}$ is the Young's modulus, Poisson ratio, and thickness of the shell). The distributed forces and moments referred to the middle surface will be taken as $p_{i}(\tau)$ and $m(\tau)$

$$
\begin{gathered}
p_{i}(\tau)=\int_{h_{-}}^{h_{+}} \vartheta(\tau, \xi) \widehat{f}_{i}(\tau, \xi) d \xi+\vartheta\left(\tau, h_{+}\right) \widehat{T}_{i+}(\tau)-\vartheta\left(\tau, h_{-}\right) \widehat{T}_{i-}(\tau), \\
m(\tau)=\int_{h_{-}}^{h_{+}} \vartheta(\tau, \xi) \xi k_{i}^{0}(\tau) \widehat{f}_{i}(\tau, \xi) d \xi+\vartheta\left(\tau, h_{+}\right) h_{+}(\tau) \widehat{T}_{i+}(\tau) k_{i}^{0}(\tau)-\vartheta\left(\tau, h_{-}\right) h_{-}(\tau) \widehat{T}_{i}(\tau) k_{i}^{0}(\tau)
\end{gathered}
$$

2. BOUNDARY VALUE PROBLEMS FOR PLATES WITH ONE-DIMENSIONAL ELASTIC REINFORCEMENTS

Let us consider a finite or infinite simply connected or nonsimply connected homogeneous linearly elastic plate $\mathrm{D}$ of constant thickness $\mathrm{H}_{0}$ reinforced along a certain part $\mathrm{L}_{\mathrm{b}}$ - of the 
boundary $\partial D$ by edge rods (stringers) with axes $L_{b}$ while there are also still internal rods with axes $L_{W}$ in elongated cavities with edges $L_{W \pm}$. The total number (connected parts) of stringers equals $N$. Let $u_{i}(x), \sigma_{i j}(x)$ be the mean displacement and stress with respect to thickness in the plate. Then the Lamé equations

$$
\left(\Lambda^{*}+\mu\right) u_{j, j i}+\mu u_{i, j j}=0
$$

are valid for a plate in the plane stress state $\left(\Lambda^{*}=2 \Lambda \mu /(\Lambda+2 \mu), \Lambda\right.$ and $\mu$ are Lamé constants).

Let us write down the boundary conditions for these equations generated by the rod reinforcements. Force transmission from the stringer to the plate can be described by different methods $[1,2]$. For definiteness, we consider that it occurs along the line of intersection of the plate middle plane and the rod side surface (infinitely thin plate) that is at a distance $h(\tau)$ from the rod central axis, i.e., along a line with the coordinates $x_{i}{ }^{0}(\tau)+$ $\mathrm{n}_{i}{ }^{0}(\tau) \mathrm{h}(\tau)$. Displacement continuity conditions should be satisfied on the line of contact. For the edge rod we have

$$
\left.u_{i}\left(s_{-}\right)\right|_{\partial D}=v_{i}\left(\tau, h_{-}(\tau)\right), s_{-} \in L_{b^{-}} .
$$

Analogously for the inner rod

$$
\left.u_{i}\left(S_{ \pm}\right)\right|_{\partial D}=v_{i}\left(\tau, h_{ \pm}(\tau)\right), s_{ \pm} \in L_{10 \pm} .
$$

Here $s_{ \pm}$is the parametrization of the rod and plate lines of contact associated with the parametrization $\tau$ of the rod middle line $L_{b}, L_{W}$ (and in agreement with it to the accuracy of a sign for a zero thickness rod); the sign + refers to quantities on the right, and - on the left of the line of rod contact with the plate (during a positive traversal); $h_{+}(t)>0$; $h_{-}(t)<0 ; d s_{ \pm}=\mp\left[\vartheta\left(\tau, h_{ \pm}\right)\right] d \tau$.

Using (1.5) to express the displacement $v_{i}\left(\tau, h_{ \pm}\right)$of the stringer in terms of the displacement of its central axis $v_{i}{ }^{\circ}(\tau)$ and taking account of the expression of these latter in terms of $\mathrm{p}_{i}$ and $\mathrm{m}$ in the relationships (1.15) and (1.7), we obtain boundary conditions for the inner reinforcement of an elastic plate by a finite-thickness elastic rod

$$
\delta_{i \pm}\left(s_{ \pm} ; u\right):=u_{i \pm}\left(s_{ \pm}\right)-v_{i}\left(\tau, h_{ \pm} ; p^{(p)}\right)=v_{0 i}\left(\tau, h_{ \pm} ; p^{(\epsilon)}\right), \tau=\tau\left(s_{ \pm}\right) .
$$

Analogously for the edge stringer, we have condition (2.2) just for $\delta_{i-}\left(s_{-} ; u\right)$. Here $p(e)$ is the part of $p$ generated by the external forces $T_{1}, f_{1}$ as well as the tip $F_{i}(k), M^{(k)}$ and the concentrated forces and moments by means of $(1.8)$, while $p(p)$ is the part of $p$ generated by forces with which the plate acts on the stringer.

For the inner stringer

$$
\begin{gathered}
p_{i}^{(p)}(\tau)=\left[-\sigma_{i j}\left(s_{+}\right) \vartheta\left(\tau, h_{+}\right) n_{j}\left(s_{+}\right)-\sigma_{i j}\left(s_{-}\right) \vartheta\left(\tau, h_{-}\right) n_{j}\left(s_{-}\right)\right] H_{0}, \\
m^{(p)}(\tau)=\left[-\sigma_{i j}\left(s_{+}\right) \vartheta\left(\tau, h_{+}\right) h_{+} n_{j}\left(s_{+}\right)-\sigma_{i j}\left(s_{-}\right) \vartheta\left(\tau, h_{-}\right) h_{-} n_{j}\left(s_{-}\right)\right] k_{i}^{0}(\tau) H_{0},
\end{gathered}
$$

where $\sigma_{i j}\left(s_{ \pm}\right)$are the stresses in the plate on the lines of contact with the rod. We should set $\sigma_{i j}\left(s_{+}\right)=0$ in these formulas for an edge stringer. We assume that $M\left(\tau_{1} ; p(p)\right)=F_{i}\left(\tau_{1}\right.$; $p(p))=0$ for unclosed stringers [for closed stringers they are determined uniquely in $p(p)$ from conditions $(1.3)$ ]. Let us note that by virtue of $(1.13)$, unknown constants $C_{i}(k)$ ( $i=$ $1,2,3, k=1-N)$, its own for each of the $N$ stringers, enters into the variable $v_{i}\left(\tau, h_{ \pm}\right.$; $\mathrm{p}(\mathrm{p})$ ) in (2.2).

The boundary conditions (2.2) must be supplemented by the last two boundary conditions in (1.1) for $\tau=\tau_{2}$ [and on a closed stringer by the conditions (1.12)] equivalent to (1.11) and (1.12) from which we obtain on each of the stringers

$$
F_{i}\left(\tau_{2}^{(k)} ; p^{(p)}\right)+F_{i}^{(e k)}=0, M\left(\tau_{2}^{(k)} ; p^{(p)}\right)+M^{(e k)}=0, k=1-N ;
$$




$$
\begin{aligned}
& F_{i}\left(\tau_{2}^{(k)} ; p^{(p)}\right):=-\int_{\tau_{1}^{(k)}}^{\tau_{2}^{(k)}} p_{i}^{(p)}(t) d t, M\left(\tau_{2}^{(k)} ; p^{(p)}\right):= \\
& :=-\int_{\tau_{1}^{(k)}}^{\tau_{2}^{(k)}} n_{i}(\tilde{\tau}) d \tilde{\tau} \int_{\tau_{1}^{(k)}}^{\tau} p_{i}^{(p)} d t-\int_{\tau_{1}^{(k)}}^{\tau_{2}^{(k)}} m^{(p)}(t) d t \\
& F_{i}^{(e k)}:=-\int_{\tau_{1}^{(k)}}^{\tau_{2}^{(k)}} p_{i}^{(e)}(t) d t-F_{i}^{(k 1)}-F_{i}^{(k 2)}-\sum_{\alpha=1}^{N_{R k}} F_{R i}^{(k \alpha)}, \\
& M^{e k)}:=-\int_{\tau_{1}^{(k)}}^{\tau_{2}^{(k)}} n_{i}(\tau) \widetilde{d \tau} \int_{\tau_{1}^{(k)}}^{\bar{\tau}} p_{i}^{(e)}(t) d t-\int_{\tau_{1}^{(k)}}^{\tau_{2}^{(k)}} m^{(e)}(l) d t-M^{(k 1)}-M^{(k 2)}- \\
& -F_{i}^{(k 1)} y_{i}^{1}\left(\tau_{2}^{(k)}\right)-\sum_{\alpha=1}^{N_{R k}}\left\{\left[y_{i}^{1}\left(\tau_{2}^{(k)}\right)-y_{i}^{1}\left(\tau_{R}^{(h \alpha)}\right)\right] F_{R i}^{(k \alpha)}+M_{R}^{(k \alpha)}\right\},
\end{aligned}
$$

where $\tau_{2}(k)=\tau_{1}(k)+\ell(k), F_{i}(k 1)+F_{i}(k 2)=M(k 1)+M(k 2)=0$ on each of the closed stringers.

Thus, if the plate along the part $\mathrm{L}_{\mathrm{b}}$ - of the boundary $\partial D$ as well as on the internal contours $L_{\mathrm{W} \pm}$ is reinforced by $\mathrm{N}$ elastic rods with longitudinal and bending stiffnesses different from zero, then we arrive at a boundary value problem for Eqs. (2.1) with boundary conditions (2.2) on $\mathrm{L}_{\mathrm{w} \pm}, \mathrm{L}_{\mathrm{b}}$ (and corresponding conditions on the rest of the boundary $\partial D \backslash \mathrm{L}_{\mathrm{b}}$ ) as well as by conditions $(2.4)$ for the functions $u_{i}(x)$ and the constants $C_{j}(k)(j=1-3, k=$ $1-N)$. Here $p_{i}(p)=p_{i}{ }^{(p)}(\tau ; u), m(p)=m(p)(\tau ; u)$ are calculated by using (2.3). If their thicknesses are considered zero in the rod models under consideration, while the stiffnesses are different from zero, then we must set $h_{+}=h_{-}=0, s_{-}=-s_{+}=\tau$ in $(2.2)$ and $(2.3)$ and the edges $L_{w \pm}$ coincide and $m(p)=0$.

We now obtain the Green-Betti formula for the plane problem of elasticity theory for the boundary conditions $(2.2)$ and $(2.3)$. Let $B(r)(D, L)$ be a class of functions $u_{i}(x)$ satisfying the Lame equation (2.1) in $D \backslash L$ such that the set $\left\{v_{i}{ }^{0}(p(p)(u)), F_{i}(p(p)(u)), M(p(p)(u))\right\} \in$ $\mathrm{H}^{(\mathrm{r})}(\mathrm{L})$ constructed by them using (1.13), (1.15), (1.17), (2.3) the bilinear form for any two functions $u_{i}, u_{i} *$ from $B(r)(D, L)$ is bounded

$$
2\left\langle u, u^{*}\right\rangle^{(p)}(D):=\iint_{D}\left[1^{*} u_{i, i} u_{j, j}^{*}+\mu\left(u_{i, j}+u_{j, i}\right)\left(u_{i, j}^{*}+u_{j, i}^{*}\right)\right] d D
$$

and the Green-Betti formula holds in D

$$
2\left\langle u, u^{*}\right\rangle^{(p)}(D)=\int_{\partial D \cup L_{L_{i}}} u_{i}^{*}(s) \sigma_{i j}(s ; u) n_{j}(s) d s,
$$

while regularity conditions $\left|u_{i}(x)\right|<C_{1}, u_{i, j}(x)=0\left(R^{-1}\right), R^{2}=x_{i}^{2} \rightarrow \infty$ are still satisfied for the infinite domain $D$.

Let us note that the imbedding in $H^{(r)}(L)$ will be satisfied if $\sigma_{i j}(s ; u) \in L_{1}(L),(|\dot{h}|$, $|x|)<\infty$ and $\left(G, G_{1}\right) \neq 0$.

For $u_{i}, u_{i}{ }^{*} \in B^{(r)}\left(D, L_{w} \cup L_{b}\right)$ we introduce the bilinear form $\left\langle u, u^{*}\right\rangle(p r):=\langle u$, $\left.u^{*}\right\rangle(\mathrm{p})(D) \mathrm{H}_{0}+\left\langle\mathrm{v}^{0}(\mathrm{u}), \mathrm{v}^{0}\left(\mathrm{u}^{*}\right)\right\rangle(\mathrm{r})\left(\mathrm{L}_{\mathrm{b}} \cup \mathrm{L}_{\mathrm{W}}\right)$, where the bilinear form $\left\langle\mathrm{v}^{0}, \mathrm{v}^{* 0}\right\rangle(\mathrm{r})$ is given by the relationship (1.9). From (2.2), (2.3), and (2.5) we have the Green-Betti formula for a reinforced plate

$$
2\left\langle u, u^{*}\right\rangle^{(p r)}=H_{0}\left[\int_{L_{b-}} \delta_{b_{-}}\left(s_{-} ; u^{*}\right) \sigma_{i j}\left(s_{-} ; u\right) n_{j}\left(s_{-}\right) d s_{-}+\right.
$$




$$
\begin{aligned}
+\int_{L_{w-}} \delta_{i-}\left(s_{-} ; u^{*}\right) \sigma_{i j}\left(s_{-} ; u\right) n_{j}\left(s_{-}\right) d s_{-}+\int_{L_{w+}} \delta_{i+}\left(s_{+} ; u^{*}\right) \sigma_{i j}\left(s_{+} ; u\right) \times \\
\left.\quad \times n_{j}\left(s_{+}\right) d s_{+}+\int_{\partial D \backslash L_{b_{-}}} u_{i}^{*}(s) \sigma_{i j}(s ; u) n_{j}(s) d s\right]+ \\
\quad+\sum_{k=1}^{X}\left[v_{i}\left(\tau_{2}^{(k)} ; p^{(p)}\left(u^{*}\right)\right) F_{i}\left(\tau_{2}^{(k)} ; p^{(p)}(u)\right)-\right. \\
\left.\quad-\dot{v}_{i}^{0}\left(\tau_{2}^{(k)} ; p^{(p)}(u)\right) n_{i}\left(\tau_{2}^{(k)}\right) M\left(\tau_{2}^{(k)} ; p^{(p)}(u)\right)\right] .
\end{aligned}
$$

We call the problem for (2.1) in which the elastic reinforcement conditions (2.2) and (2.3) are given on the inner contours $L_{w \pm}$ and the part $L_{b}$ - of the boundary, the forces

$$
\sigma_{i j}(s ; u) n_{j}(s)=g_{i}(s), s \in L_{T},
$$

are given on the part $\mathrm{L}_{\mathrm{T}}$ of the boundary, and the displacements

$$
u_{i}(s)=f_{i}(s), s \in L_{u}
$$

on the part $L_{u}$, the problem $(r-T-u)$. If the sections $L_{u}=\theta$ or $L_{T}=\theta$ or conditions of elastic one-dimensional reinforcement are given on the whole boundary $\partial \mathrm{D}$, then we arrive at the problems, $(r-T),(r-u)$, and $(r)$, respectively.

Taking into account that $\langle\mathrm{u}, \mathrm{u}\rangle \mathrm{pr} \geq 0$, we obtain the following uniqueness theorem in the class $B(r)$ ( $\left.D, L_{b} \cup L_{W}\right)$ from (2.8). The solution of the problems $(r)$ and $(r-T)$ is unique to the accuracy of a rigid shift $u_{c i}(x)=v_{c i}(x)=C_{i}{ }^{0}+C_{3}{ }^{0} e_{i j} x_{j}(x \in D)$ in a finite domain $D$ and to the accuracy of a rigid shift without rotation $u_{c i}(x)=v_{C i}(x)=c_{i}{ }^{0}(x \in D)$ in the infinite domain $D$. The constants $C_{j}(k 0)=C_{j}{ }^{0}(j=1-3, k=1-N)$ in (1.13) for each connected part of the reinforcement. The solution of the problems $(r-u)$ and $(r-T-u)$ is unique in both the finite and the infinite domain $D$.

Let us note that sufficiency of the conditions $(2.2)-(2.4),(2.9),(2.10)$ hence follows for uniqueness of the determination of not only $u_{i}$ and $v_{0 i}$ but also the constants $C_{i}(k)$ on all rods $\left[C_{j}\left(k_{0}\right)=C_{j}{ }^{0}\right.$ to $v_{C i}$ accuracy in the problems $(r)$ and $\left.(r-T)\right]$.

Setting $u_{i} *(x)=v_{i} *=C_{i}+C_{3} e_{i j} x_{j}\left(x_{j} \in D\right)$ in (2.8) $\left(C_{3}=0\right.$ for the infinite domain $D)$, and taking account of (2.6), we have that it is necessary for the solution of the problems $(r)$ and $(r-T)$ to exist in the class $B(r)\left(D, L_{b} \cup L_{W}\right)$ that the principal vector, and for a finite domain the principal moment of the external forces applied to the reinforced body as well, be equal to zero.

Up to now we spoke in this section about the problem of the plane state of stress for a plate reinforced by a rod (stringer). But it is easy to see that all the assertions obtained here are also carried over to the plane strain problem for a cylinder reinforced by a cylindrical shell if $\Lambda^{*}$ is replaced by $\Lambda$ in (1.1) and (2.7) and we set $H_{0}=1$ in (2.8) and take account of the remark at the end of Sec. 1 .

\section{STRESS SINGULARITY AT ANGULAR POINTS AND ENDS OF STRINGERS}

We assume in analyzing the singularities that the stresses near the singular points in the plate satisfy the estimate $\sigma_{i j}(r)=0\left(r^{-\gamma}\right), \gamma<1$. Hence, taking account of (1.13), (1.14), and (2.3), there.results that if the stiffnesses $G(s)$ and $G_{1}(s)$ at the point $s_{*}$ under investigation are not zero while the curvature $\chi(s)$ is bounded, then $\left|\dot{v}_{i}\left(s_{\dot{k}}, p(p), m(p)\right)\right|$, $\left[n_{i} \dot{v}_{i}\left(s_{*} p(p), m(p)\right)\right]^{\prime} \mid<\infty$. Then components with these terms can be carried over into the right side of $(2.2)$ and we arrive at the problems

$$
u_{i}\left(s_{-}\right)=v_{i}\left(\tau, h_{-}\right), s_{-} \in L_{b_{-}^{-}}, u_{ \pm i}\left(s_{ \pm}\right)=v_{i}\left(\tau, h_{ \pm}\right), s_{ \pm} \in L_{w \pm},
$$

where the right sides $v_{i}\left(\tau, h_{ \pm}\right)$are conditionally continuous while their derivatives are bounded at $s_{*}$ and Holder-like in the left and right neighborhoods of $s_{*}$ if the functions $x(\tau), \vartheta\left(\tau, h_{ \pm}(\tau)\right), 1 / G(\tau), 1 / G_{1}(\tau)$ possess this property. Furthermore, we denote the parameters $s_{ \pm}, h_{ \pm}$by $s$ and $h$ if $s_{*} \in L_{ \pm}$, respectively, $\tau_{*}=\tau\left(s_{*}\right)$. But conditions (3.I) are the 
boundary conditions of the first boundary value problem (according to Kupradze) and the stress singularity has been studied well in such problems (see [10, 11] and their bibliography). Let $\omega$ be an interior angle of the contour $L_{b}$ or $L_{W \pm}$ at the point $s_{*}$. If $\pi<\omega<$ $2 \pi$, then the displacements and stresses in a partial local coordinate system $(r, \theta)$ with origin at $s_{*}$ and angle $\theta$ measured counterclockwise from the bisectrix of $\omega$ have the form

$$
\begin{gathered}
u_{i}(\rho, \theta)=C_{i}+\sum_{m=1}^{2} K_{m} u_{i}^{(m)}(\theta) \rho^{1-\gamma_{m}}+u_{i}^{0}(\theta) \rho+u_{i}^{*}(\rho, \theta), \\
\sigma_{i j}(\rho, \theta)=\sum_{m=1}^{2} 2 \mu K_{m} \sigma_{i j}^{(m)}(\theta) \rho^{-\gamma_{m}^{\mathrm{II}}}+\sigma_{i j}^{0}(\theta)+\sigma_{i j}^{*}(\rho, \theta) .
\end{gathered}
$$

Here the degrees of the stress singularities $\gamma_{1} I_{(\omega)}, \gamma_{2} \operatorname{II}(\omega) \in(0,1)$ are roots of the equations $\Delta_{*}\left(x, \omega, \gamma_{1} I I\right)=0$ and $\Delta_{*}\left(-x, \omega, \gamma_{2} I I\right)=0$, respectively, where the function $\Delta_{*}(x$, $\omega, \gamma):=x \sin [(\gamma-1) \omega]+(\gamma-1) \sin \omega$ and the plane elasticity theory constant $x=\left(\Lambda^{*}+\right.$ $3 \mu) /\left(\Lambda^{*}+\mu\right)$ for the plane state of stress and $x=(\Lambda+3 \mu) /(\Lambda+\mu)$ for plane strain. The stress intensity factors $K_{m}$ depend on the geometry and given loads and should be determined from the solution of the problem as a whole. The eigenfunctions $u_{i}(\mathrm{~m})(\theta), \sigma_{i j}(\mathrm{~m})(\theta)$ depend only on the aperture angle and are written down explicitly $u_{i}^{*}(\rho, \theta), u_{i j} *(\rho, \theta), \sigma_{i j} *(\rho$, $\theta) \rightarrow 0$ as $\rho \rightarrow 0$ while the functions $u_{i}{ }^{0}(\theta), \sigma_{i j}{ }^{0}(\theta)$ bounded for $\pi<\omega<2 \pi$ (as can be shown, say, by using methods of [12]) have the form

$$
\begin{gathered}
u_{\rho}^{0}:=-\left(B_{2}-B_{4} \operatorname{ctg} \omega\right) \cos 2 \theta-\left(B_{1} \operatorname{ctg} \omega+B_{3}\right) \sin 2 \theta-B_{4} / \sin \omega, \\
u_{\theta}^{0}:=\left(B_{2}-B_{4} \operatorname{ctg} \omega\right) \sin 2 \theta-\left(B_{1} \operatorname{ctg} \omega+B_{3}\right) \sin 2 \theta+B_{1} / \sin \omega, \\
\left.\sigma_{\rho \rho}^{0}:=-\left(B_{2}-B_{4} \operatorname{ctg} \omega\right) \cos 2 \theta-\left(B_{1} \operatorname{ctg} \omega+B_{3}\right) \sin 2 \theta-2 B_{4} /(x-1) \sin \omega\right], \\
\left.\sigma_{\theta \theta}^{0}:=\left(B_{2}-B_{4} \operatorname{ctg} \omega\right) \cos 2 \theta+\left(B_{1} \operatorname{ctg} \omega+B_{3}\right) \sin 2 \theta-2 B_{4} /(x-1) \sin \omega\right], \\
\sigma_{\rho \theta}^{0}:=\left(B_{2}-B_{4} \operatorname{ctg} \omega\right) \sin 2 \theta-\left(B_{1} \operatorname{ctg} \omega+B_{3}\right) \cos 2 \theta ; \\
B_{1}:=u_{1, s}^{s}\left(s_{*}\right) \cos (\omega / 2)+u_{2, s}^{A}\left(s_{*}\right) \sin (\omega / 2), \\
B_{2}:=-u_{1, s}^{A}\left(s_{*}\right) \cos (\omega / 2)-u_{2, s}^{s}\left(s_{*}\right) \sin (\omega / 2), \\
B_{3}:=u_{1, s}^{s}\left(s_{*}\right) \sin (\omega / 2)-u_{2, s}^{A}\left(s_{*}\right) \cos (\omega / 2), \\
B_{4}:=-u_{1, s}^{A}\left(s_{*}\right) \sin (\omega / 2)+u_{2, s}^{s}\left(s_{*}\right) \cos (\omega / 2), \\
u_{i, s}^{s}\left(s_{*}\right)=\left[v_{i, s}\left(\tau\left(s_{*}\right)\right)\right]^{s}, u_{i, s}^{A}\left(s_{*}\right)=\left[v_{i, s}\left(\tau\left(s_{*}\right)\right)\right]^{A} .
\end{gathered}
$$

Here and henceforth, the notation is $\varphi^{s}\left(s_{*}\right):=\left[\varphi\left(s_{*}+0\right)+\varphi\left(s_{*}-0\right)\right] / 2, \varphi^{A}\left(s_{*}\right):=\left[\varphi\left(s_{*}+()\right)-\varphi\left(s_{*}-\right.\right.$ $0)] / 2$ for the function $\varphi(s)$. Hence it is seen that in the first problem the coefficients are determined explicitly in terms of the tangential derivatives of the given boundary displacements around the angular point. Taking account of $(1.5)$ we obtain $\left[u_{i}, s_{i}\right]^{A}=-[\dot{h}(1+$ $\left.\mathrm{xh})^{-1} \mathrm{u}_{\mathrm{i}, \mathrm{s}} \mathrm{k}_{\mathrm{i}}\right]^{A}$ from the last condition $(1.4)$ in the problem under consideration with given elastic reinforcements, i.e.,

$$
\begin{gathered}
B_{2} \sin \omega-B_{4} \cos \omega=[\dot{h}(1+\chi h)]^{A}\left(B_{2} \cos \omega-B_{4} \sin \omega\right)- \\
-\left[\dot{h} /\left(1+\chi \chi^{h}\right)\right]^{s}\left(B_{1} \cos \omega+B_{3} \sin \omega\right) .
\end{gathered}
$$

Therefore, $B_{2}$ can be expressed in terms of the remaining coefficients $B_{1}, B_{3}, B_{4}$, which, like $\mathrm{K}_{\mathrm{m}}$, are not determined a priori. Let us note that if $\dot{h}\left(\tau_{*}\right)=0$, in particular, if the rod has zero thickness, then because of (3.5) the first parentheses in each of the expressions (3.3) equal zero while if $\hat{h}=0$ and $\omega=\pi / 2$, then the tangential stress is $\sigma_{s n}=0$.

In the case when $\omega=\pi$, i.e., $s_{*}$ is a point of smoothness of the contour, the asymptotic (3.2) and (3.3) is replaced by the following

$$
\begin{gathered}
u_{\rho}(\rho, \theta)=C_{\rho}(\theta)+\left\{\left[-(x+1)(\pi x)^{-1} B_{1}(\ln \rho-1)+K_{01}\right] \sin 2 \theta+\right. \\
\left.+K_{02}(\cos 2 \theta+1)-B_{1}(\pi x)^{-1} \theta[(x+1) \cos 2 \theta-x+1]+B_{2}\right\} \rho+u_{\rho}^{*}(\rho, \theta), \\
u_{\theta}(\rho, \theta)=C_{\theta}(\theta)+\left\{\left[-(x+1)(\pi x)^{-1} B_{1}(\ln \rho-1)+K_{01}\right](\cos 2 \theta-1)-\right. \\
\left.-K_{02} \sin 2 \theta+B_{1}(\pi x)^{-1}(x+1) \theta \sin 2 \theta+B_{3}\right\} \rho+u_{\theta}^{*}(\rho, \theta),
\end{gathered}
$$




$$
\begin{gathered}
\sigma_{\rho \rho}=2 \mu\left\{\left[-(x+1)(\pi x)^{-1} B_{1} \ln \rho+K_{01}\right] \sin 2 \theta+\right. \\
+K_{02}[\cos 2 \theta+2 /(x-1)]+\theta(\pi x)^{-1} B_{1}[-(x+1) \cos 2 \theta+2]+ \\
\left.+2 B_{2} /(x-1)\right\}+\sigma_{\rho \rho}^{*}, \\
\sigma_{\theta \theta}=2 \mu\left\{-\left[-(x-1)(\pi x)^{-1} B_{1} \ln \rho+K_{01}\right] \sin 2 \theta+\right. \\
+K_{02}[-\cos 2 \theta+2 /(x-1)]+\theta(\pi x)^{-1} B_{1}[(x+1) \cos 2 \theta+2]+ \\
\left.+2 B_{2} /(x-1)\right\}+\sigma_{\theta \theta}^{*}, \\
\sigma_{\rho \theta}=2 \mu\left\{\left[-(x+1)(\pi x)^{-1} B_{1} \ln \rho+K_{01}\right] \cos 2 \theta+K_{02} \sin 2 \theta+\right. \\
\left.+\theta(\pi x)^{-1} B_{1}(x+1) \sin 2 \theta-(\pi x)^{-1} B_{1}\right\}+\sigma_{\rho \theta}^{*} .
\end{gathered}
$$

Here and henceforth $C_{\rho}(\theta), C_{\theta}(\theta)$ is the constant shift $C_{j}$ in Cartesian coordinates expressed in polar coordinates, for simplicity it is assumed that $h\left(s_{s_{k}}\right)=0$ for $\omega=\pi$, then $B_{4}=0$ because of (3.5). The remaining coefficients $B_{1}$, just as the factors $K_{01}, K_{02}$, are generally not determined a priori. However, if we use the notation $x_{h}(\tau):=x(\tau)+G(\tau) h(\tau) /\left[G_{1}(\tau) \vartheta(\tau\right.$, h)], then for $\omega=\pi$ we find $F_{i} k_{i}{ }^{0}(\tau)+\chi_{h}(\tau) M_{R}=G(\tau) u_{i, s}(s) k_{i}(s)$ from $(1.5)$ and $(1.6)$. Then taking account of $(1.4)$ and $(3.4)$

$$
B_{1}=\left[F_{R i}^{*} k_{i}^{0}\left(\tau_{*}\right)+\gamma_{h}^{s}\left(\tau_{*}\right) M_{R}^{*}-2 \gamma_{h}^{A}\left(\tau_{*}\right) M^{s}\left(\tau_{*}\right)+2 G^{A}\left(\tau_{*}\right) B_{3}\right] /\left[2 G^{s}\left(\tau_{*}\right)\right]
$$

where $\mathrm{F}_{\mathrm{Ri}} *$ and $\mathrm{M}_{\mathrm{R}}$ * are concentrated external forces and moments at the point $\tau_{*}$. It is hence seen that for $\dot{h}\left(s_{*}\right)=0$ the logarithmic terms in (3.6) can occur when there is a concentrated longitudinal force or moment at the point of smoothness $s_{*}$ or a jump in the longitudinal stiffness $G$ or in $\chi_{h}$. If the longitudinal stiffness and $\chi_{h}$ are continuous, the coefficient $B_{1}$ of the logarithm is expressed explicitly in terms of the given concentrated force and moment and equal zero if they are missing at $\tau_{*}$.

If the angle is $0<\omega<\pi$ at the point $s_{*}$ then the asymptotics (3.2) and (3.3) hold with (3.5) taken into account but for $K_{1}=K_{2}=0$ terms with these exponents can be combined with $u_{i}{ }^{*}, \sigma_{i j}{ }^{*}$ since $\gamma_{1}{ }^{I I}, \gamma_{2}{ }^{I I}<0$. Therefore, the principle parts of the asymptotics are given by $(3.3)$.

If $G=G_{1}=\infty$, then from $(1.5)$ and $(1.6)$ it is easily seen that $u_{i, s}=c_{2} n_{1}$. Then $B_{2}=$ $B_{4}=B_{1} \cos \omega+B_{3} \sin \omega=0$ and in place of (3.3) for $\omega \neq \pi$ we obtain $u_{\rho}{ }^{0}=\sigma_{\rho \rho}{ }^{0}=\sigma_{\rho \theta} \theta^{0}=0$, $u_{\theta}{ }^{0}=B_{1} / \sin \omega$. For $\omega=\pi$ it is necessary to substitute relationships $B_{1}=B_{2}=B_{4}=0$ in the asymptotic (3.6).

The asymptotics (3.2), (3.3), (3.6) indeed refer to an analysis of the tip domains of internal stringers with nonzero thickness if it is assumed that the stringer model being used is applicable at these points also. Then $w$ are the angles of the local geometry of the tip domains. If the internal stringer thickness in the tip domain equals zero then $\omega=2 \pi, \gamma_{1}=$ $\gamma_{2}=1 / 2$ in this domain. As before the asymptotics of the displacements and stresses have the form (3.2) for $\omega=2 \pi$ but the smallest terms $u_{i}{ }^{0}, \sigma_{i j}{ }^{0}$ will here differ from (3.3). If $s_{*}$ is the point where contact between the edge stringer and the plate terminates and, moreover, displacements are given on the boundary, then interchanges of the type of boundary conditions of the asymptotics $(3.2)-(3.4),(3.6),(3.7)$ are conserved completely at this point.

By analogy with the above, it is easy to see that at the point of interchange of the type of boundary conditions occurring at the end of the edge stringer when forces are later given on the boundary (conditions of the second problem), the asymptotic will be the same as in the neighborhood of the point of interchange of the type of boundary conditions of the first and second problems ( and curvature of the stringer if the stiffnesses are $G\left(s_{*}\right), G_{1}\left(s_{*}\right) \neq 0$ while $\chi\left(s_{*}\right) \neq \infty$ at the points $s_{*}$ being investigated.

Therefore, in problems with one-dimensional elastic reinforcements having nonzero longitudinal and bending stiffnesses and bounded curvature, the principal terms of the asymptotics, and particularly, the degrees of the singularities will be the same as in the problem with given displacements. The influence of the stiffnesses and curvature affects only the values of the coefficients of this asymptotic.

Let us note that the case when $G(s), G_{1}(s) \rightarrow 0, \chi(s) \rightarrow \infty$ as $s \rightarrow s^{*}$ can be investigated by methods similar to those used in $[13,14]$. 
For rods (shells) of nonzero thickness in zones with large curvature, including in the neighborhood of angular point of the axis, there occurs an intersection of the normals to it in the bulk of the rod. For a correct formulation of the problem it is necessary to refine the theory of rods in such zones. In particular, these zones can be considered rigid inserts, i.e., $G=G_{1}=\infty$ can be set there. To remove given and contact forces distributed over the rigid zone on the axis, (1.8) and (2.3) can be replaced by any relationships yielding their principal vector and moment in this zone. Then the results obtained above are conserved even for such cases.

\section{LITERATURE CITED}

1. Mookey and Sternberg, "Load transmission from an edge stiffness rib to a sheet (revision of a Melan problem), Trans. ASME, Ser. E., Mechanics, 34, No. 3.

2. Mookey and Sternberg, "Load transmission from a transverse tensile rod to a semi-infinite elastic plate," Trans. ASME, Ser. E., Mechanics, 35, No. 4.

3. M. P. Sheremet'ev, Plates with Reinforced Edges [in Russian], Izd. L'vov Univ., L'vov (1960).

4. E. I. Mikhailovskii, Direct, Reverse, and Optimal Problems for Shells with Reinforced Edge [in Russian], Izd. Leningr. Univ., Leningrad (1986).

5. S. K. Kanaun, "Thin defect in a homogeneous elastic medium," Izv. Akad. Nauk SSSR, Mekh. Tverd. Tela, No. 3 (1984).

6. N. F. Morozov, S. A. Nazarov, and A. V. Proskura, "Boundary value problems of elasticity theory for plane domains with thin flanges," in: Mechanics of a Deformable Body, [in Russian], Inst. Probl. Mekh. Akad. Nauk SSSR (1986).

7. G. N. Savin and V. I. Tul'chii, Plates Reinforced by Composite Rings and Elastic Straps [in Russian], Naukova Dumka, Kiev (1971).

8. S. E. Mikhailov and I. V. Namestnikova, "Plane problems for nonsimply connected elastically reinforced plates," in: Mechanics of Inhomogeneous Structures. Abstract of Reports, 2nd All-Union Conf., [in Russian], Vo1. 2 (1987).

9. 0. B. Golubev, "Generalization of the theory of thin rods," Trudy, Leningr. Politekh. Inst. No. 226 (1963).

10. V. Z. Parton and P. I. Perlin, Methods of Mathematical Elasticity Theory [in Russian], Nauka, Moscow (1981).

11. M. L. Williams, "Stress singularities resulting from various boundary conditions in angular corners of plates in extension," J. App1. Mech., 19, No. 4 (1952).

12. S. E. Mikhailov, "Asymptotics of solutions of certain integral equations and plane elasticity theory problems near angles for forces given on the boundary," Izv. Akad. Nauk SSSR, Mekh. Tverd. Tela, No. 3 (1989).

13. 0. V. Sotkilava and G. P. Cherepanov, "Certain problems of inhomogeneous elasticity theory," Prik1. Mat. Mekh., 38, No. 3 (1974).

14. N. Kh. Arutyunyan and S. A. Nazarov, "Singularities of stress functions at angular points of the transverse section of a twisted rod with a thin reinforcing coating," Prik1. Mat. Mekh. 47, No. 1 (1983). 